\title{
USO ETNOBOTÁNICO Y PRINCIPIOS ACTIVOS DE Monnina crassifolia KUNTH; POLYGALACEAE
}

\author{
ETHNOBOTANICAL USE AND ACTIVE INGREDIENTS \\ OF Monnina crassifolia KUNTH; POLYGALACEAE
}

\section{Paúl Hidalgo ${ }^{1}$, María López ${ }^{2 *}$, Moraima Mera², Luz Cañamar ${ }^{2}$ \& Omar Malagón ${ }^{1}$}

\author{
Recibido: 28 de diciembre 2019 / Aceptado: 19 de junio 2020 \\ Publicado en línea: 29 de junio 2020 \\ DOI: $10.26807 /$ ia.v8i2.128
}
Palabras clave: cromatografía, etnobotánica, metabolitos, valor de importancia

Keywords: chromatography, ethnobotany, metabolites, importance value

\section{RESUMEN}

Ecuador es uno de los países con mayor diversidad biológica y cultural, la etnobotánica representa una aproximación al uso y manejo de los recursos naturales; los usos medicinales juntamente con el alimenticio juegan un papel

1 Universidad Técnica Particular de Loja. Departamento de Química y Ciencias Exactas. Loja, Ecuador. (pahidalgo@utpl.edu.ec; omalagon@utpl.edu.ec)

2 Pontificia Universidad Católica del Ecuador Sede Ibarra. Escuela de Ciencias Agrícolas y Ambientales. Ibarra, Ecuador. (*correspondencia: mflopez2@pucesi.edu.ec; mmera@pucesi.edu.ec; Icanamar@pucesi.edu.ec) 
primordial en el bienestar de la población rural. Los estudios etnobotánicos ayudan a identificar, cómo se relacionan e influyen las plantas en el desarrollo de las culturas, tal es el caso de la Comunidad de Angochagua en donde se utiliza la medicina ancestral como un modo de vida. El objetivo de esta investigación fue conocer el índice de valor de uso, conocimiento relativo de la especie y el nivel de uso significativo de las especies botánicas en las comunidades de Angochagua, Provincia de Imbabura. Una vez establecida la especie más vulnerable en cuanto a la pérdida del uso ancestral Monnina crassifolia Kunth, se utilizaron técnicas cromatográficas para el aislamiento e identificación de los metabolitos secundarios teniendo como resultado una posible saponina triterpenoídica esterificada con ácido benzoico. Las sustancias aisladas fueron probadas frente a bacterias Gram + y Gram -, al igual que frente a los sistemas enzimáticos MAO-A y MAO-B sin mostrar una actividad biológica significativa.

\section{ABSTRACT}

Ecuador is one of the countries with the greatest biological and cultural diversity, ethnobotany represents an approach to the use and management of natural resources; medicinal uses together with food uses play a major role in the wellbeing of the rural population. Ethnobotanical studies help to identify how plants are related and influence the development of cultures; such is the case of the Angochagua Community where ancestral medicine is used as a way of life. The objective of this investigation was to know the index of use value, relative knowledge of the species and the level of significant use of botanical species in the communities of Angochagua, Imbabura Province. Once the most vulnerable species in terms of loss of ancestral use Monnina crassifolia Kunth was established, chromatographic techniques were used for the isolation and identification of secondary metabolites, resulting in a possible triterpenoid saponin esterified with benzoic acid. The isolated substances were tested against Gram + and Gram - bacteria, as well as against the MAO-A and MAO-B enzyme systems without showing significant biological activity. 


\section{INTRODUCCIÓN}

El uso etnobotánico busca el conocimiento ancestral y el saber botánico tradicional, relacionado al uso de las especies vegetales, siendo un pilar fundamental en países en vía de desarrollo ya que les proporciona una alternativa en la asistencia médica.

Las plantas medicinales cumplen una función importante en el sistema de salud de comunidades indígenas, ya que son usadas de manera frecuente por la mayoría de sus pobladores, Ecuador posee una alta diversidad biológica y cultural por lo que se encuentra descrito como uno de los países con un gran potencial en el uso tradicional de las especies vegetales, ya que estas, proporcionan beneficios curativos en diferentes comunidades del país, tal es el caso de la Parroquia de Angochagua en la cual se mantienen rasgos ancestrales en el manejo y utilización de la medicina tradicional.

En Ecuador, la etnobotánica es una herramienta útil para el rescate y revalorización del conocimiento ancestral sobre el uso del recurso vegetal, además es el campo cientí- fico que estudia las interacciones que se establecen entre el hombre y las plantas a través del tiempo. En Ecuador, los pocos estudios de plantas medicinales y etnobotánica se han desarrollado en la zona andina enumerando las plantas que conoce $y$ usa la gente de las comunidades indígenas.

El uso de la medicina tradicional en el tratamiento de enfermedades, es una práctica que se ha llevado a cabo desde tiempos ancestrales y ha demostrado que es una de las mejores opciones beneficiando a las personas y comunidades que mantienen y conservan el uso de plantas medicinales, caso particular es el área de estudio la Parroquia de Angochagua donde se utiliza este recurso, manteniendo su valor y uso cultural.

La capacidad económica en las comunidades indígenas es reducida, siendo difícil acceder a los medicamentos modernos por lo que es más fácil utilizar la medicina tradicional mediante el uso de sustancias derivadas de las plantas medicinales. 
El conocimiento ancestral de la medicina tradicional en el área rural de la Parroquia de Angochagua puede estar disminuyendo con el paso del tiempo, debido al salto generacional de su población y a la existencia de un menor interés o preocupación por parte de los jóvenes de mantener estos valores ancestrales, tal es el caso de la especie Monnina crassifolia, la cual ha sido utilizada para la limpieza capilar evitando la caída del cabello, canas y caspa, lamentablemente esta especie ha disminuido el uso en los jóvenes de la Parroquia de Angochagua.

El género Monnina pertenece a la familia Polygalacea que comprende 21 géneros y alrededor de 800-1000 especies distribuidas en las regiones tropicales y subtropicales a nivel mundial (Eriksen \& Persson, 2007). Existe una gran variedad de metabolitos secundarios presentes en la familia Polygalaceae. De acuerdo a investigaciones fitoquímicas realizadas, los productos químicos predominantes son las saponinas, xantonas y oligosacáridos esterificados (Lepore et al., 2011).

En la actualidad la especie $M$ crassfolia no ha sido estudiada y no se han encontrado reportes sobre metabolitos secundarios aislados a partir de esta especie, sin embargo se ha tomado como referencia el estudio de otras especies del mismo género, principalmente de $M$ sylvatica en donde se ha comprobado la actividad antifúngica de bifenilos y xantonas en el extracto lipofilico de las raíces (Bashir, Hamburger, Gupta, Solis, \& Hostettmann, 1992), además del género $M$ obtusifolia su extracto de $\mathrm{n}-\mathrm{BuOH}$ ha demostrado que sus hojas inhiben los factores de crecimiento endotelial vascular (VEGF -A) o la interacción con el factor de crecimiento placentario con Flt- 1 (VEGF receptor 1) (Lepore et al., 2011).

Por lo antes mencionado, el objetivo del presente estudio es establecer el estado actual del conocimiento ancestral en cuanto al uso de plantas medicinales en especial de la especie Monnina crassifolia en la Parroquia de Angochagua, Provincia de Imbabura, Ecuador, así como también aislar e identificar metabolitos secundarios por medio de técnicas cromatográficas y espectroscópicas, para contribuir con el estudio para la conser vación y aprovechamiento de especies vegetales de interés medicinal en el Ecuador. 


\section{MATERIALES Y MÉTODOS}

\section{Trabajo de campo}

Para el estudio etnobotánico, se llevaron a cabo conversaciones con habitantes de la comunidad rural para informarlos acerca de los objetivos del estudio.

Para la determinación del tamaño de la muestra de informantes se empleó la cartográfica temática del Instituto Geográfico Militar (IGM) a una escala 1:50000 con referencia a capas de delimitación y geo distribución de las viviendas dentro de la comunidad ubicando un total de 225 viviendas a visitar.

Para la selección de las viviendas a encuestar, se utilizó el método del centroide y distribución sistemática a través de la generación de una rejilla de 1000 m x 1000 m, donde se seleccionaron las cercanas a la intercepción de los ejes, sumando un total de 45 domicilios a muestrear que corresponde al 19,5\% dentro del área rural.

Se obtuvo una muestra representada por 22 hombres y 37 mujeres, con conocimientos sobre plantas medicinales. El rango de edad de los informantes hombres fue de 15 a 85 años, mientras que las mujeres estuvieron en un rango de edad de 21 a 78 años. Se aplicaron entrevistas semiestructuradas en las que se indagó sobre las plantas empleadas para tratar alguna enfermedad, los usos medicinales tradicionales, métodos de preparación, vía de administración y partes de la planta. Además, se obtuvo información sobre aspectos socioculturales tales como la edad y sexo.

Se utilizó una prueba de Chi-cuadrado $(\chi 2)$ para determinar las diferencias significativas en la importancia de las partes de las plantas usadas por la comunidad (hojas, tallo, raíz, flor, fruto, cristal, corteza, planta completa), el tipo de preparación (infusión, emplasto, jugo, cocción, macerado, triturado), la vía de administración (bebida, comida, baños, uso externos), y el conocimiento de las especies por rangos de edad (rango I: entre 15 y 35 años, $\mathrm{n}=25$; rango II: entre 36 y 85 años, $n=25$ ) y sexo de las personas entrevistadas. 


\section{Colecta de muestra botánica}

Para la colecta, secado, deshidratado y almacenamiento de la muestra se utilizaron los protocolos del Herbario de la PUCE-SI:

a) Recolección: Se realizaron tres colectas de corteza de raíz de Monnina crassifolia en la Comunidad de Angochagua, localizada coordenadas geográficas $0^{\circ} 16^{\prime} 47^{\prime \prime}$ de latitud Sur y $78^{\circ} 02^{\prime} 10^{\prime \prime}$.
La Comunidad de Angochagua posee una población total al 2015 de 3017 Habitantes, con una extensión de 12392 ha $\left(123 \mathrm{~km}^{2}\right)$, sus límites son al norte: Ibarra y La Esperanza, cantón Ibarra, provincia de Imbabura, al sur: parroquia Olmedo, cantón Cayambe, provincia de Pichincha. Al este: parroquia San Pablo del Lago y González Suárez, cantón Otavalo, provincia de Imbabura.

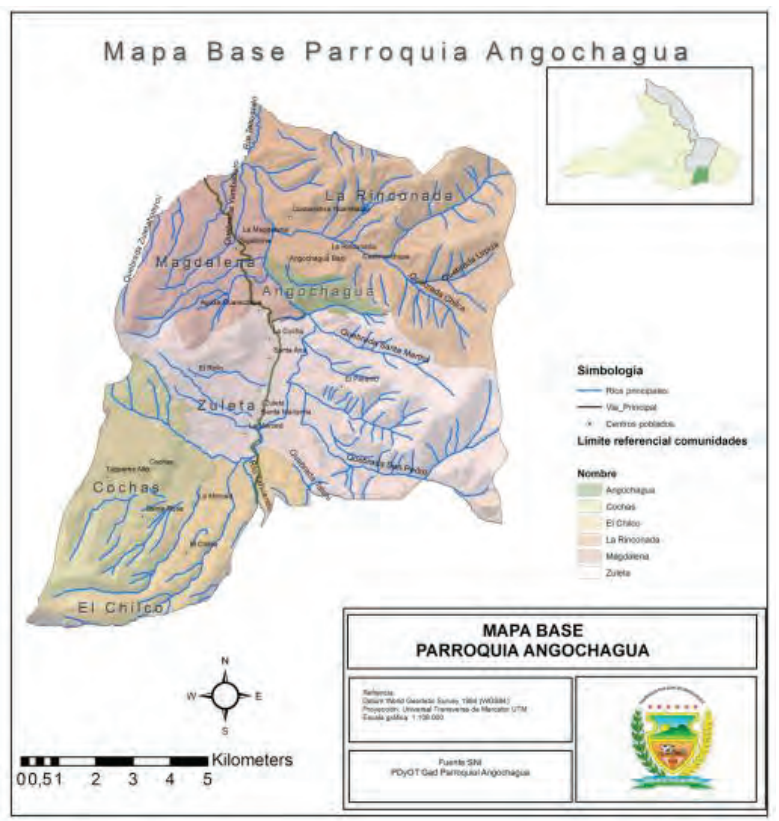

Figura 1. Mapa de Ubicación de la Comunidad de Angochagua.

(Sandoval, 2017) 
b) Secado: las muestras en el Herbario de la PUCE-SI se desinfectaron rociando alcohol potable al $96 \%$, luego se prensó la planta en papel periódico, papel filtro y tablas triplex. A continuación, se envolvió el paquete prensado en funda plástica para ingresar a cuarentena en el cuarto frío por un período de 48 horas $\mathrm{a}-10^{\circ} \mathrm{C}$.

c) Deshidratado: Transcurrida la cuarentena se procedió a secar la planta por cuatro horas aproximadamente a $30{ }^{\circ} \mathrm{C}$, tomando en cuenta que las hojas debían presentar una textura suave al terminar el secado. La humedad determinada en la planta fue de $78,37 \%$.

Una vez separada cada parte de la muestra se sometió al deshidratador de marca TERMOKOOL por 20 minutos.

d) Almacenamiento: Finalmente se pulverizó la muestra vegetal en un triturador de marca RETSCHGM 200 durante un minuto a 4500 revoluciones por minuto, resultando un peso de $270 \mathrm{~g}$.

\section{Análisis de datos etnobotánicos.}

La información etnobotánica proporcionada por los habitantes del área de estudio, fue organizada en una base de datos empleando una hoja de cálculo de Microsoft Office Excel 2018.

Los porcentajes y frecuencias de las citaciones de las plantas medicinales asociadas al conocimiento tradicional, fueron utilizados para el análisis etnobotánico. Se emplearon 3 índices para determinar la importancia de las diferentes especies identificadas en el área de estudio.

\section{* Índice de valor de uso de especies.} Este índice expresa la importancia o valor cultural de una especie determinada para todos los informantes entrevistados. Para estimar el índice de valor de uso general de cada especie para todos los informantes (IVUs) se utilizó la fórmula:

$$
I V U s=\frac{\sum i V U i s}{N S}
$$

Donde: $\mathrm{VUis}=$ valor de uso de la especie por cada informante, y Ns es el número de informantes para cada especie. 
* Conocimiento relativo de la especie por varios encuestados (RVU). Se utilizó la siguiente formula:

$$
R V U=\frac{\sum \frac{V U i s}{I V U S}}{N s p}
$$

Donde: $\mathrm{VUis}=$ valor de uso de la especie por cada informante, IVUs = es el índice de valor de uso de la especie, y Nsp = es el número de especies.

* Nivel de uso significativo Tramil (UST). Para estimar el nivel de uso significativo para cada especie y verificar su aceptación cultural, se utilizó la metodología propuesta por (Germosén, 1995).

Esta metodología, expresa que aqueIlos usos medicinales que son citados con una frecuencia superior o igual al $20 \%$, por las personas encuestadas que usan plantas como primer recurso para un determinado problema de salud, pueden considerarse significativos desde el punto de vista de su aceptación cultural y, por lo tanto, merecen su evaluación y validación científica. El UST se calcula mediante la siguiente ecuación:

$$
U S T=\frac{U \text { so especie }(s)}{N i s} \times 100
$$

Donde: Uso especie $(\mathrm{s})=$ número de citaciones para cada especie, y Nis = número de informantes encuestados.

\section{Obtención de extractos}

A partir de 250 g de la raíz de Monnina crassifolia, se procedió a macerar por triplicado con disolventes de polaridad creciente, iniciando con éter etílico durante 5 horas con 1000 $\mathrm{mL}$ de solvente, seguido de acetato de etilo, metanol y agua.

Posteriormente se filtró al vacío y se concentró el extracto por rotaevoparación a presión reducida. El extracto acuoso se concentró mediante el equipo de liofilización LABCONCO serie 77608 .

\section{Separación por partición del ex- tracto metanólico}

A partir de $5 \mathrm{~g}$ de extracto metanólico se efectuó la partición con n-butanol y agua destilada relación (1:1) efectuando tres lavados, que fueron concentrados en rotaevoparación a 
presión reducida, obteniendo la fase de n-butanol 3,84 g mientras que la fase de agua un total de 1,2 g.

\section{Fraccionamiento del extracto meta- nólico (fase butanólica)}

Del extracto butanólico obtenido se efectuó el fraccionamiento por cromatografía en columna, en relación 1:100 tomando $3,84 \mathrm{~g}$ de extracto con 385 g de sílica gel 60 (Merck 0.0015-0040 mm). La fase móvil utilizada se preparó con disolventes en orden de polaridad creciente iniciando con acetato de etilo-metanolagua 90:10:2,5, disminuyendo el acetato de etilo paulatinamente hasta Ilegar a 60:10:2,5 y finalmente metanol al $100 \%$, obteniendo un total de 391 fracciones de $8 \mathrm{~mL}$.

\section{Cromatografía en capa fina}

Se realizó cromatografía en capa fina (TLC, por sus siglas en inglés) de todas las fracciones obtenidas de la columna cromatográfica, utilizando placas de sílica gel 60 F254 (fase directa). Los disolventes para la fase móvil fueron acetato de etilo-metanol-agua en relación 8:1:0,25 (v/v), para la unión de acuerdo a la separa- ción y similitud de compuestos, la visualización se la realizó en luz UV 254 y 365 nm, y reveladas con ácido sulfúrico al $5 \%$ y vainillina. Posteriormente se unieron las fracciones con igual factor de retención (Rf).

\section{Purificación de la fracción MC028}

La purificación de la fracción MC028 fue a partir de 111,8 mg, en columna cromatográfica con $11 \mathrm{~g}$ de sílica gel 60 (Merck 0,0015-0040 mm) en relación 1:100, utilizando como disolvente en relación acetato de etilometanol-agua 7:1,3:1.

\section{Determinación de la actividad anti- microbiana (CMI) de las fracciones obtenidas}

La determinación de la concentración mínima inhibitoria de las dos fracciones obtenidas se realizó por medio de la técnica de microdilución en caldo, utilizando cinco bacterias Gram-negativas: Escherichia coli (ATCC 25922), Salmonella tiphymurium (LT2), Proteus vulgaris (ATCC 8427), Pseudomonas aeruginosa (ATCC 8427), Klebsiella pneumoniae (ATCC 9997), y una bacteria Gram-positiva: Staphylococcus aureus (ATCC 28185). 
Ensayo de inhibición de monoamina oxidasa (MAO) de las fracciones obtenidas

Las soluciones mitocondriales (MAOA y $\mathrm{MAO}-\mathrm{B})$ se suspenden en PBS y DMSO a diferentes concentraciones, se dividen en pequeñas alícuotas y almacenan a una temperatura de -80 ${ }^{\circ} \mathrm{C}$. Se trabajó con $1 \mathrm{mg}$ en las dos muestras a una solución de 10 $\mathrm{mg} / \mathrm{mL}$ de DMSO.
El procedimiento para la medición de la actividad selectiva de MAO-A y MAO-B consiste en la adición de las siguientes soluciones:

Vehículo (DMSO + molécula) + Fracción mitocondrial (MAO-A y MAOB) + Sustrato no selectivo (Kynuramine) + CLO (Inhibidor selectiva MAO-A) o DEP (Inhibidor selectivo $M A O-B)$.

\section{RESULTADOS}

\section{Inventario etnobotánico}

En el área estudiada se obtuvo registros de un total de 75 especies de plantas medicinales identificadas hasta nivel de especie, distribuidas en 22 familias y 32 géneros.

Las familias con mayor número de especies medicinales en todo el estudio fueron Asteraceae, seguida por Lamiaceae, Apiaceae, Crassulaceae, Fabaceae, Poaceae y Rutaceae, finalmente la Poligalaceae.

En cuanto a la forma de obtención del recurso vegetal la mayoría de encuestados (92\%) acceden a las plan- tas medicinales mediante cultivos en huertos caseros, y el resto de entrevistados acceden a estas a través de la compra en mercados locales.

\section{Plantas utilizadas para la medicina}

Después del análisis de las encuestas realizadas en la comunidad de Angochagua se evidenció que las hojas son las estructuras más utilizadas en la preparación de la medicina, representando el $80,7 \%$ del total de menciones, seguido por la utilización del tallo $(11,0 \%)$, la raíz $(4,0 \%)$, toda la planta $(2,7 \%)$, flores, cristales, corteza y fruto (1,6\% cada uno). La prueba de $\chi 2$ muestra que existen di- 
ferencias significativas en la importancia de las partes usadas por la comunidad $(X 2$ cal $=350,5, X 2$ th $=$ $13,07, g l=7, p<0,001)$.

\section{Clasificación de formas de creci- miento vegetal}

Se pudo evidenciar que del total de plantas medicinales mencionadas $(\mathrm{n}=75)$, las hierbas representas por 48 especies (64\%), mientras que los árboles, arbustos, y epífitos están representados por $14(18,66 \%), 8$ $(10,66 \%)$ y $5(6,66 \%)$ especies respectivamente.

\section{Vía de administración más empleada}

La vía de administración más empleada es la bebida con un 79,0 \%, seguida del uso externo con un 10,3 $\%$, la comida con $8,0 \%$ y el baño con $2,7 \%$.

El análisis de la prueba de $\chi 2$ muestra que existen diferencias significativas en las vías de administración de las plantas medicinales por la comunidad (X2 cal $=160,80, X 2$ th $=6,90$, gl $=2,9 \mathrm{p}<0,001)$.

\section{Conocimiento de las especies de acuerdo al sexo y rango de edad de los informantes}

En relación con la información recopilada de acuerdo al sexo de las personas de la comunidad encuestadas se registró un total de 64 especies mencionadas por las mujeres y 60 especies por los hombres. La prueba de $\chi 2$ muestra que no existen diferencias significativas en el conocimiento de hombre y mujeres en el número de plantas de uso medicinales conocidas (X2 cal =0,001, X2 th $=2,74$, $\mathrm{gl}=1, \mathrm{p}=1,0)$.

Por rangos de edad se encontró que las personas más jóvenes (rango I) conocen 22 especies de uso medicinal, una menor cantidad que las personas de mayor edad (rango 2) que conocen 53 especies. La prueba de $\chi 2$ muestra que no existen diferencias significativas en el conocimiento entre los dos rangos de edad en el número de plantas de uso medicinales conocidas (X2 cal =0,373, X2 th $=3,84, \mathrm{gl}=1, \mathrm{p}=0,541)$. 
Preparación, vía de administración y categorías de uso de las plantas medicinales

Las formas de preparación para el uso de las plantas medicinales en la comunidad de Angochagua son variadas, la mayoría de las preparaciones son realizadas en forma de infusión (80,5\%), seguido del triturado con un porcentaje de $(11,0 \%)$ y en forma de emplasto, jugo, cocción y macerado con un porcentaje de 9,3\% cada una (Figura 3).

La prueba de $\chi 2$ muestra que existen diferencias significativas en las formas de preparación de las plantas medicinales por la comunidad (X2 $\mathrm{cal}=2898,15, \mathrm{X} 2$ th $=10,08, \mathrm{gl}=4$, $p<0,001)$. En relación con la información recopilada las personas de la comunidad de Angochagua utilizan principalmente plantas frescas en el momento del preparado.

\section{Conocimiento relativo de las espe- cies por los informantes}

El índice de conocimiento relativo de las especies por varios encuestados (RVU, por sus siglas en inglés) y el nivel de uso significativo (UST, por sus siglas en inglés) permitieron identificar la importancia de cada una de las 75 especies de plantas medicinales reportadas para esta investigación.

Las especies más importantes para los pobladores de la Comunidad de Angochagua de acuerdo a los índices antes mencionados fueron: la hierba luisa (Cymbopogon citratus; RVU: 0.65; UST: $53 \%$ ), el orégano (Origanum vulgare; RVU:0,60; UST: 50 \%), la hierba buena (Mentha sativa; RVU: 0.56; UST: $48 \%$ ), la menta (Mentha rotundifolia; RVU:0,45; UST: 38 \%), el toronjil (Melissa officinalis; RVU: 0,30; UST: $32 \%$ ).

Entre las especies importantes en conocimientos ancestrales y que han perdido su uso tradicional se tiene al Ivilán (Monnina crassifolia; RVU: 0,15; UST: $10 \%$ ), siendo esta la especie a estudiar en cuanto a sus principios activos para revalorizar su conocimiento ancestral.

\section{Descripción morfológica y taxonó- mica de la Monnina crassifolia}

La familia Polygalaceae es utilizada en la medicina herbal, como expec- 
torante estimulante, diurético y diaforético. Debido a un alto contenido de saponinas y propiedades antifúngicas, varias especies de Monnina a menudo se han utilizado como champú anticaspa en América tropical (Eriksen \& Persson, 2007). Como ejemplo, la especie africana Securidaca longepedunculata, pertenciente a la familia Polygalaceae, tiene hasta 100 propiedades médicas, destacando principalmente la inhibición selectiva de la replicación del VIH (Mahmood et al., 1993).

El género Monnina perteneciente a la subfamilia Polygaleae fue bautizado por los botánicos españoles Ruiz y Pavón sobre un grupo de plantas de Perú y Chile (Ruiz, López, \& Pavón, 1798). Para la descripción del género Monnina se tomó como referencia a especies descritas por la revista del Instituto Botánico Darwinion en Argentina, detallando al género como plantas con hojas simples, alternas, brevemente pecioladas. Inflorescencia en racimo simple, espiciforme. Flores pequeñas, blanco-amarillentas, o rosado-verdosas, con cinco sépalos de prefloración quincuncial, dos externos, uno interno y dos laterales. Fruto una sámara 1-2 semi- nada, generalmente reticulada, a lada o sin alas, glabra o pubescente. Raíz generalmente perpendicular, ramificada y cilíndrica de color castaño oscuro o claro, marrón oscura y amariIlo. Semillas piriformes, comprimidas o no, con tegumento tenue y delgado rostro en el ápice (Grondona, 1945).

Este género es únicamente americano y se extiende desde el Sur de Estados Unidos hasta el centro de Chile y la Plata. En el Ecuador el género Monnina tiene especies arbustivas que comprende unas 150 especies. Se encuentran unas 32 variedades, mejor representadas en la zona andina; sobre los $2400 \mathrm{~m}$ se han registrado 24 especies arbustivas.

Existe una gran variedad de usos en el género, dentro del cual cabe recalcar la especie Monnina obtusifolia, la cual se utiliza en la medicina tradicional de Ecuador como un antifúngico, antitumoral, antiséptico, antiperiodontitis y limpiador de piel (Pinto, Fuzzati, Chiriboga, \& Hostettmann, 1994).

Monnina crassifolia es un arbusto que mide hasta 1,5 m de alto. Las hojas son alternas, lanceoladas, miden 
hasta $6 \mathrm{~cm}$ de largo, siendo gruesas. La inflorescencia tiene numerosas flores, miden hasta $6 \mathrm{~cm}$ de largo, tienen la forma de mariposa, de color azul o azul-morado, uno de los pétalos Ilamado "quilla" tiene la punta amarilla. Los frutos son carnosos, con forma de elipse o de fréjol, miden hasta $8 \mathrm{~mm}$ de largo, de color vino morado y verde en la base cuando están inmaduros, de color negro-morado cuando están maduros (Eriksen, 1993).

Tiene uso medicinal como antibacteriano al utilizarse como jabón y medioambiental debido a que la planta se usa como cerca viva o para hacer leña.

\section{Principios activos de Monnina cras- sifolia}

Luego de realizar un screening fitoquímico de la muestra en estudio (corteza de la raíz del Ivilán), se obtuvieron resultados positivos para saponinas, triterpenos y aceites fijos. Los resultados negativos fueron para: Alcaloides, taninos, flavonoides, esteroides y aceites esenciales.

\section{Tabla 1. Screening fitoquímico}

\begin{tabular}{ll}
\hline Alcaloides & Negativo \\
Saponinas & Positivo \\
Taninos & Negativo \\
Flavonoides & Negativo \\
Triterpenos & Positivo \\
Esteroides & Negativo \\
Aceites Fijos & Positivo \\
Aceites esenciales & Negativo \\
\hline
\end{tabular}

El estudio de interés por la aplicación ancestral de la raíz de Ivilán, se centró en el contenido de Saponinas, por lo que se realizó una prueba semicuantitativa de su contenido.

Tabla 2. Determinación semi-cuantitativa del contenido de saponinas en la corteza de raíz de Ivilán

\begin{tabular}{lcccc}
\hline Muestra & Corresponde & Masa (g) & Altura $(\mathrm{cm})$ & $\begin{array}{c}\text { Concentración } \\
\text { de Saponinas (\%) }\end{array}$ \\
\hline M1R1 & Colecta 1 & 0,5034 & 3,5 & 0,4 \\
M1R2 & Colecta 1 & 0,5033 & 4,0 & 0,5 \\
M2R1 & Colecta 2 & 0,4983 & 6,7 & 0,6 \\
M2R2 & Colecta 2 & 0,4964 & 6,7 & 0,8 \\
\hline
\end{tabular}


De las muestras analizadas la concentración media fue del 0,6 \% aproximadamente

\section{Extractos obtenidos de la especie Monnina crassifolia}

En la Tabla 3 se describen los pesos y rendimientos obtenidos del extracto de la raíz con los solventes éter etílico, acetato de etilo, metanol y agua.

Tabla 3. Rendimiento de los extractos.

\begin{tabular}{lccc}
\hline Extracto & $\begin{array}{c}\text { Peso inicial } \\
\text { planta seca }(g)\end{array}$ & $\begin{array}{c}\text { Peso final } \\
(\mathrm{g})\end{array}$ & $\begin{array}{c}\text { Rendimiento } \\
(\%)\end{array}$ \\
\hline Éter etílico & & 0,663 & 0,27 \\
Acetato de etilo & 250 & 2,328 & 0,94 \\
Metanol & & 30,167 & 12,07 \\
Agua & & 3,181 & 1,27 \\
\hline
\end{tabular}

Fuente: (Hidalgo, 2017)

Mediante TLC en fase directa de los extractos obtenidos, representados en la Figura 2, se observó la abundancia de los diferentes compuestos presentes en la especie.

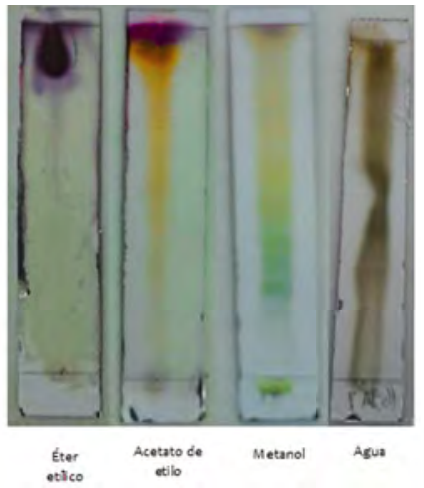

Figura 2. TLC de los diferentes compuestos presentes en la especie. (Hidalgo, 2017) 
Fraccionamiento del extracto de metanol tras, las cuales se unificaron según criterios del factor de retención (Rf) en

Se fraccionaron $3,84 \mathrm{~g}$ del extracto TLC dando un total de 30 fracciones metanólico obteniéndose 392 mues(MC001-MC030) descritas en la Tabla 4.

Tabla 4. Fraccionamiento cromatográfico del extracto total de metanol.

\begin{tabular}{|c|c|c|c|c|}
\hline Fracciones & Muestras & Proporción & $\begin{array}{l}\text { Mezcla de } \\
\text { disolvente }\end{array}$ & $\begin{array}{l}\text { Peso } \\
\text { (mg) }\end{array}$ \\
\hline MC001 & 0 & \multirow{13}{*}{$9: 1: 0,25$} & \multirow{13}{*}{$\begin{array}{c}\text { AcOEt- } \\
\mathrm{MeOH}- \\
\mathrm{H}_{2} \mathrm{O}\end{array}$} & 8,3 \\
\hline MC002 & 1 a 5 & & & 5,8 \\
\hline MC003 & 6 a 13 & & & 40,80 \\
\hline MC004 & 16 a 22 & & & 41,40 \\
\hline MC005 & 23 a 40 & & & 59,10 \\
\hline MC006 & 41 a 58 & & & 386,10 \\
\hline MC007 & 59 a 66 & & & 25,40 \\
\hline MC008 & 67 a 75 & & & 33,10 \\
\hline MC009 & 76 a 84 & & & 34,10 \\
\hline MC010 & 85 a 89 & & & 26,40 \\
\hline MC011 & 90 a 99 & & & 63,70 \\
\hline MC012 & 100,00 & & & 5,90 \\
\hline MC013 & 101 a 106 & & & 35,80 \\
\hline MCO14 & 107 a 128 & \multirow{4}{*}{$8: 1: 0,25$} & \multirow{4}{*}{$\begin{array}{c}\text { AcOEt- } \\
\mathrm{MeOH}- \\
\mathrm{H}_{2} \mathrm{O}\end{array}$} & 116,00 \\
\hline MC015 & 129 a 136 & & & 27,70 \\
\hline MC016 & 137 a 164 & & & 90,30 \\
\hline MC017 & 165 a 181 & & & 48,20 \\
\hline MC018 & 182 a 213 & \multirow{4}{*}{$7: 1: 0,25$} & \multirow{4}{*}{$\begin{array}{c}\text { AcOEt- } \\
\text { MeOH- } \\
\mathrm{H}_{2} \mathrm{O}\end{array}$} & 84,3 \\
\hline MC019 & 214 a 229 & & & 12,30 \\
\hline MCO20 & 230 a 256 & & & 18,00 \\
\hline MC021 & 257 a 264 & & & 44,40 \\
\hline MC022 & 265 a 276 & \multirow{8}{*}{$6: 1: 0,25$} & \multirow{8}{*}{$\begin{array}{c}\text { AcOEt- } \\
\mathrm{MeOH}- \\
\mathrm{H}_{2} \mathrm{O}\end{array}$} & 10,50 \\
\hline MC023 & 277 a 284 & & & 18,4 \\
\hline MC024 & 285 a 328 & & & 10,9 \\
\hline MC025 & 329 a 337 & & & 80,5 \\
\hline MC026 & 338 a 363 & & & 8,5 \\
\hline MC027 & 364 a 372 & & & 16,5 \\
\hline MC028 & 373 a 379 & & & 111,8 \\
\hline MC029 & 380 a 391 & & & 24,6 \\
\hline MC030 & 392 & 1 & $\mathrm{MeOH}$ & 69,8 \\
\hline
\end{tabular}

Fuente: (Hidalgo, 2017) 
La cromatografía en capa fina (TLC) de metabolitos secundarios de las 30 se realizó utilizando como fase móvil fracciones obtenidas (Figuras 3 y 4), la mezcla acetato de etilo-metanol- para su posterior purificación. agua $8: 1: 1$, observándose la riqueza

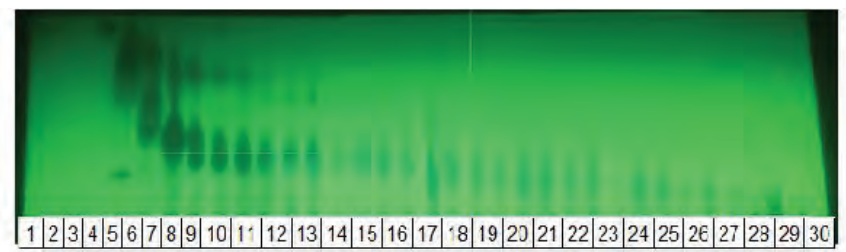

Figura 3. TLC luz UV 254 nm

(Hidalgo, 2017)

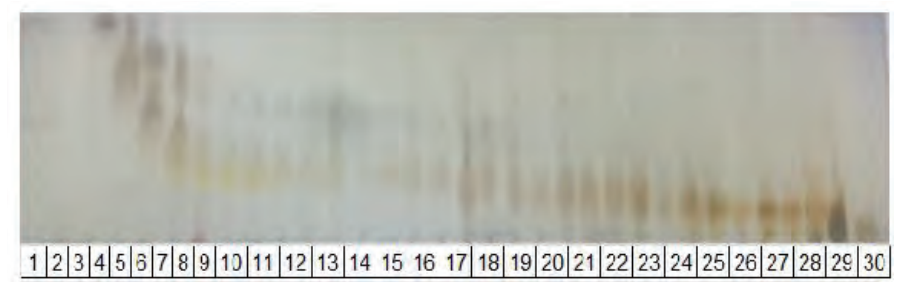

Figura 4. TLC revelada en relación 8:1:0,25 acetato de etilo-metanol-agua

(Hidalgo, 2017)

\section{Fracción MC028}

Por medio de una microcolumna cromatográfica se efectuó la purificación de la fracción MC028 (111,8 mg) en $11 \mathrm{~g}$ de sílica gel en relación acetato de etilo-metanol-agua 7:1,3:1.
Del fraccionamiento se obtuvieron 49 muestras que luego se unieron de acuerdo al perfil TLC y Rf dando un total de 8 fracciones descritas a continuación: 
Tabla 5. Separación cromatográfica de la fracción MC028

\begin{tabular}{lcccc}
\hline Fracciones & Muestras & Proporción & $\begin{array}{c}\text { Mezcla de } \\
\text { disolvente }\end{array}$ & $\begin{array}{c}\text { Peso } \\
\text { (mg) }\end{array}$ \\
\hline F28-1 & $0-6$ & & & 1,7 \\
F28-2 & 7 & & & 1,4 \\
F28-3 & 8 & & AcOEt- & 5,6 \\
F28-4 & $9-10$ & $7: 1,3: 1$ & $\mathrm{MeOH}-$ & 15 \\
F28-5 & 11 & & $\mathrm{H}_{2} \mathrm{O}$ & 5,2 \\
F28-6 & $12-13$ & & & 6,4 \\
F28-7 & $14-48$ & & & 14,2 \\
F28-8 & 49 & & & 6,3 \\
\hline
\end{tabular}

Fuente: (Hidalgo, 2017

Cromatografía preparativa de la fracción F28-4

A través de TLC preparativa fase reversa (RP-18), utilizando una placa con una dimensión de $20 \times 10 \mathrm{~cm}$, se realizó la siembra de la muestra F284 de 15 mg, siendo la fase móvil metanol: agua 9:1. El revelado se realizó con la ayuda de la luz UV $254 \mathrm{~nm}$ y 360 nm, observando una mancha de color azul oscuro y un color morado (Figura 5 y 6), las cuales fueron raspadas y recuperado el metabolito adsorbido.

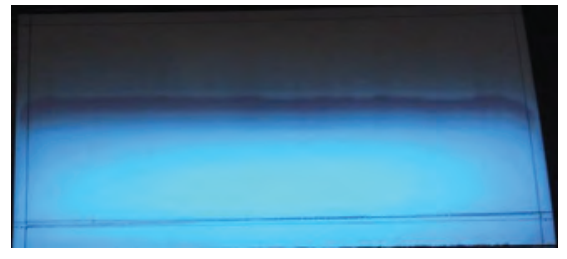

Figura 5. TLC luz UV $254 \mathrm{~nm}$ (Hidalgo, 2017)

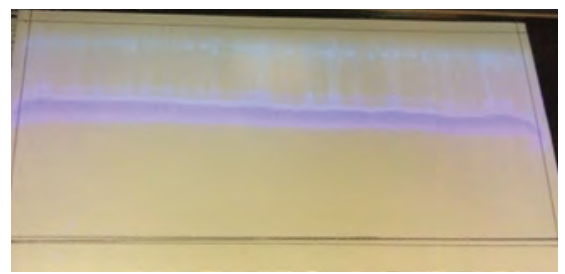

Figura 6. TLC luz UV $365 \mathrm{~nm}$ (Hidalgo, 2017) 
Se obtuvieron dos fracciones (MC028-03 y MC028-04) con peso de 3,8 mg y 2,4 mg, se procedió a realizar TLC fase directa en relación 7:1,3;1 acetato de etilo-metanolagua, en sílica gel de $9 \mathrm{~cm}$ de longitud.

Observando en la luz UV de 365 nm un color azul brillante, que luego al revelarla con ácido sulfúrico al $5 \%$ y vainillina, se observó un color amarillo intenso, presentando $\mathrm{Rf}$ de 0,38 y 0,35 .

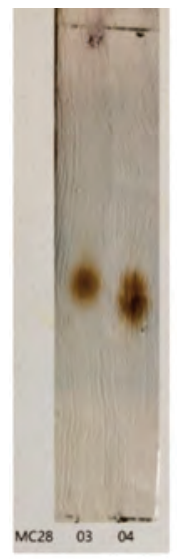

Figura 7. TLC revelada $M C 028-03$ y MC028-04

(Hidalgo, 2017)

Posteriormente se realizó el análisis por elucidación química de las fracciones MC028-03 y MC028-04 en
RMN en un equipo Varian $\mathrm{N}^{\circ}$ de serie 21953 operando a $400 \mathrm{MHz}$ para ${ }^{1} \mathrm{H}$ y $100 \mathrm{MHz}$ para ${ }^{13} \mathrm{C}$. Usando como disolvente metanol deuterado $\left(\mathrm{CD}_{3} \mathrm{OD}\right)$.

\section{Resonancia Magnética Nuclear de las fracciones obtenidas.}

Mediante microcolumna con polaridad acetato de etilo-metanol-agua $(7: 1,3: 1)$ de la fracción 373 a 379 se obtuvieron $15 \mathrm{mg}$, por medio de TLC preparativa fase reversa, se obtuvieron dos fracciones de peso $3,8 \mathrm{mg}$ y $2,4 \mathrm{mg}$, con un color blanco, y solubles en metanol.

Los espectros de ${ }^{1} \mathrm{H}$ y ${ }^{13} \mathrm{C}$ de la fracción MC028-03 tienen similitud con la fracción MC028-04, considerándose que la fracción MC028-04 está compuesta por una fracción de la sustancia presente en MC028-03 y otra sustancia desconocida. Por tal motivo, en el presente trabajo se analizó únicamente el espectro de ${ }^{1} \mathrm{H}$ y ${ }^{13} \mathrm{C}$ de la fracción MC028-03, que asemeja una fracción más pura.

Por tanto, se establece la presencia de un benzoato (Figura 8), en la estructura molecular de la fracción MC028-03. 


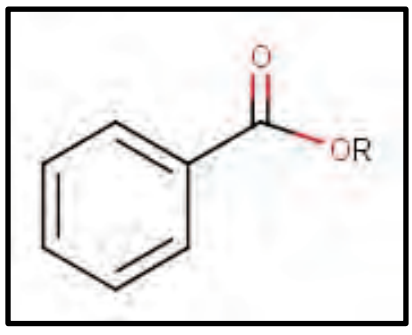

Figura 8. Estructura molecular del Benzoato

Se realizó una interpretación del espectro obtenido de las fracciones MC028-03 por RMN en un equipo Varian $\mathrm{N}^{\circ}$ de serie 21953 operando a $100 \mathrm{MHz}$ para ${ }^{13} \mathrm{C}$ disuelta en metanol deuterado $\left(\mathrm{CD}_{3} \mathrm{OD}\right)$, identificando lo siguiente:

De acuerdo al análisis del espectro obtenido de ${ }^{13} \mathrm{C}$, se observan picos con valores altos correspondientes a la estructura carbonílica del benzoato y acetato en 175 y 172 ppm, así mismo la presencia de un grupo carbonilo en 216,18, que se ubica presumiblemente en el carbono 3 de un triterpeno

Al observar el espectro en un rango de 129,6 a 130,8 , se aprecian los picos correspondientes a 4 de los carbonos aromáticos, que se identifican con los carbonos de la unidad de ácido benzoico. En el resto de la fracción estudiada se observan la posible presencia de los carbonos olefínicos y los carbonos aromáticos restantes. A campos entre 35 y 108 se pueden observar los carbonos que presumiblemente pertenecen al esqueleto triterpénico y a los azúcares, incluídos los anoméricos a campos más bajos.

Finalmente, a campos altos (15 - 30 ppm) se encuentran los picos de los radicales metilo pertenecientes a una posible estructura terpénica. La pequeña cantidad de sustancia obtenida no permitió realizar estudios espectrales más profundos.

\section{Ensayo para la determinación de la actividad antimicrobiana de las frac- ciones MC028-03 y MC028-04}

Se emplearon las cinco bacterias Gram-negativas: Escherichia coli, Salmonella tiphymurium, Proteus vulgaris, Pseudomonas aeruginosa, KlebsieIla pneumoniae, y una bacteria Gram-positiva: Staphylococcus aureus. Tomando como referencia el peso de las fracciones se trabajó con una disolución 10 veces la proporción con Dimetilsulfóxido (DMSO), $1 \mathrm{mg} / 100 u \mathrm{~L}$ DMSO, siendo la más alta en el ensayo de $250 \mathrm{ug} / \mathrm{mL}$, obteniendo los resultados mostrados en la Tabla 6. 
Tabla 6. Concentración mínima inhibitoria (CMI) de las fracciones F1 y F2

\begin{tabular}{|c|c|c|}
\hline \multirow{2}{*}{$\begin{array}{l}\text { Bacterias Gram - y Gram + } \\
\text { ATB (Ec. S.t y S.a 3,906 } \mu \mathrm{g} / \mathrm{mL} \text {; P.v y } \\
\text { P.a } 15,625 \mu \mathrm{g} / \mathrm{mL} ; \text { K.p } 1,953 \mu \mathrm{g} / \mathrm{mL} \text { ) }\end{array}$} & \multicolumn{2}{|c|}{$\begin{array}{c}\text { CMI de las fracciones } \\
\text { obtenidas }\end{array}$} \\
\hline & F1 & F2 \\
\hline Escherichia coli (Ec.) & NA & NA \\
\hline Salmonella tiphymurium (S.t) & NA & NA \\
\hline Proteus vulgaris (P.v) & NA & NA \\
\hline Pseudomonas aeruginosa (P.a) & NA & NA \\
\hline Klebsiella pneumoniae (K.p) & NA & NA \\
\hline Staphylococcus aureus (S.a) & NA & NA \\
\hline
\end{tabular}

(NA): No activo, a la dosis más alta probada

Fuente: (Hidalgo, 2017)

Ensayo para la determinación de la Los compuestos ensayados fueron las actividad de inhibición de monoa- fracciones MC028-03 y MC028-04 mina oxidasa (MAO) en las fraccio- en una solución de DMSO de 10 nes MC028-03 y MC028-04. $\mathrm{mg} / \mathrm{mL}$, obteniendo los resultados mostrados en la Tabla 7.

Tabla 7. Concentraciones inhibidoras de MAO-A y MAO-B

\begin{tabular}{lcccc}
\hline $\begin{array}{l}\text { Endpoint 316-357, 0- } \\
\text { 177.5min, 50ug/ml }\end{array}$ & Promedio & $\begin{array}{c}\text { Forma } \\
\text { estándar }\end{array}$ & $\begin{array}{c}\text { \% actividad } \\
\text { residual MAO-B }\end{array}$ & $\begin{array}{c}\text { \% actividad } \\
\text { residual MAO-A }\end{array}$ \\
\hline $\begin{array}{l}\text { DMSO vs MAO-A 0\% - } \\
\text { MAO-B 0\% (+CLO+DEP) }\end{array}$ & $1 \%$ & $0 \%$ & 0 & 0 \\
\hline DMSO vs MAO-A+B & $100 \%$ & $3 \%$ & 100 & 100 \\
\hline DMSO vs MAO-B (+CLO 500nM) & $54 \%$ & $2 \%$ & 100 & 0 \\
\hline DMSO vs MAO-A (+DEP 1000nM) & $42 \%$ & $1 \%$ & 0 & 100 \\
\hline MC28-03 vs MAO-A+B & $97 \%$ & $3 \%$ & & 93 \\
\hline MC28-03 vs MAO-B & $50 \%$ & $1 \%$ & 93 & 89 \\
\hline MC28-03 vs MAO-A & $39 \%$ & $1 \%$ & & \\
\hline MC28-04 vs MAO-A+B & $97 \%$ & $3 \%$ & & \\
\hline MC28-04 vs MAO-B 04 vs MAO-A & $49 \%$ & $1 \%$ & & \\
\hline
\end{tabular}

Fuente: (Hidalgo, 2017) 
El ensayo demostró que no hay inhibición sobre MAO-A y MAO-B de las fracciones obtenidas MC28-03 y MC28-04.

\section{DISCUSIÓN}

Las plantas han sido la base de los sistemas de medicina tradicional que han existido desde hace miles de años. Incluso en los tiempos modernos, los sistemas basados en plantas siguen desempeñando un papel esencial en el cuidado de la salud (Chivian, 2002).

De acuerdo a la Organización Mundial de la Salud (OMS), el $11 \%$ de fármacos básicos y esenciales son exclusivamente de origen vegetal obtenidos a partir de precursores naturales (Rates, 2001).

Según la misma organización, alrededor del 65-80 \% de la población mundial en los países en desarrollo, debido a la pobreza y la falta de acceso a la medicina moderna, depende esencialmente de las plantas para su atención primaria de salud. Sin embargo, algunas plantas han sido estudiadas científicamente para la evaluación de su calidad, seguridad y eficacia (Calixto, 2005).
América Latina, ofrece una gran diversidad de plantas y cultivos estacionales únicos debido principalmente a la presencia de áreas naturales como la cordillera de los Andes, la selva amazónica y los bosques tropicales y subtropicales de América Central. Varios informes científicos han señalado el potencial terapéutico de ciertas plantas y alimentos de esta área (Ranilla, Kwon, Apostolidis, \& Shetty, 2010).

La Comunidad de Angochagua es una de las comunidades que guarda sus conocimientos ancestrales en la Sierra Norte del Ecuador teniendo como resultado un total de 75 especies de plantas medicinales reportadas como el índice de conocimiento relativo de las especies por varios encuestados (RVU) y el nivel de uso significativo (UST) permitieron identificar la importancia de cada una de las en esta investigación.

Entre las especies importantes en conocimientos ancestrales y que han perdido su uso tradicional se tiene al 
Ivilán (Monnina crassifolia; RVU:0,15; UST: $10 \%$ ), siendo esta la especie a estudiar en cuanto a sus principios activos para revalorizar su conocimiento ancestral.

La producción de estos compuestos a menudo es baja (menos de $1 \%$ de peso seco) y depende mucho del estado fisiológico y de desarrollo de la planta (Rao \& Ravishankar, 2002). El aislamiento dirigido a los principios activos de la planta se continúa realizando en muchas áreas académicas, gubernamentales, y laboratorios. A partir de extractos que presentan una actividad biológica particular de interés se purifican estos principios por cromatografía, guiados por evaluación periódica con uno o más sistemas de bioensayo, lo que resulta en el eventual aislamiento de uno o más constituyentes biológicamente activos (Kinghorn, 1992).

La elucidación estructural realizada sobre la fracción MC28-03 permite comparar los reportes obtenidos sobre saponinas triterpénicas citotóxicas en las raíces de Symplocos chinensis, donde se ha obtenido un compuesto denominado symplocososide K, $3 \beta$-0- [( $\beta$-D-glucopyranosyl $(1-2)][(\alpha-L-$ arabinofuranosyl](1-4)]-
$\beta$-O-[(3-0-acetyl-6-methyl)-glucuronopyranosyl] -21 $\beta$-0-[(2Z)3,7-dimethyl-2,6-octadienoyl]-22 $\alpha$-0-benzolyl] -R1-barrigenol, Figura 9, este tipo de estructuras se caracterizan por la presencia de siete grupos metilos terciarios entre $\delta 0,90$ y 1,51 ppm, y un doble enlace típicas por ${ }^{13} \mathrm{C}$ en RMN a $\delta 125,8$ y 142,9 ppm, revelando olean-12-ene - triterpeno (Olugbade, Ogundaini, Birlirakis, Païs, \& Martin, 2000), la parte azucarada corresponde a tres tipos: ácido glucurónico, glucosa y arabinosa, presentes como tres protones anoméricos en $\delta$ $4,89,5,10$ y 5,88 y con la relación de ${ }^{13} \mathrm{C}-\mathrm{RMN}$ en $\delta 110,6,104,9$ y $104,8$. Finalmente, la unión de la unidad de benzoilo en C-22 de la aglicona que se representa entre el protón de $\delta$ 6,53 y el espectro de ${ }^{13} \mathrm{C}-\mathrm{RMN}$ en $\delta$ en 166,9 (Cioffi et al., 2006; Fu, Wang, Gao, Tang, \& Yu, 2005).

Sin embargo, por la poca cantidad obtenida no se puede efectuar un análisis completo de RMN tanto en ${ }^{1} \mathrm{H},{ }^{13} \mathrm{C}$, DEPT, COSY, HMBC $y$ HSQC, que permita una identificación absoluta de la estructura molecular de mezcla MC028-03. 

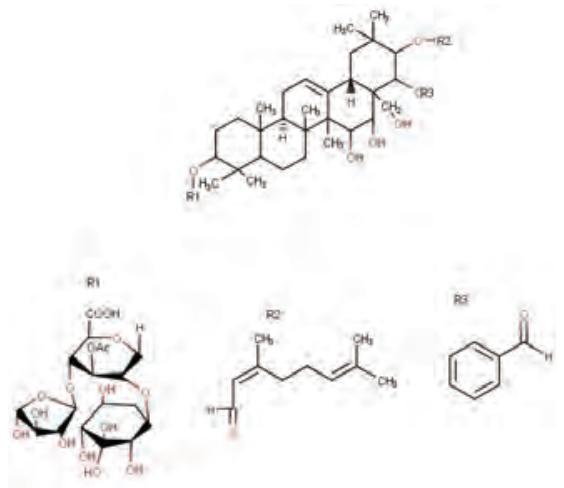

Figura 9. Estructura symplocososide K.

(Fu et al., 2005)

Dentro de la actividad biológica de algunas saponinas triterpénicas, se ha demostrado que presentan actividad citotóxica principalmente para tres líneas celulares tumorales, que comprenden J774.A1 (Monocíticas / macrófagos murinos), HEK - 293 (Células epiteliales humanas de riñón) y WEHI 164 (Células de fibrosarcoma murino) (Cioffi et al., 2006), además se le atribuye actividades antialergénicas, antibacterianas, anti-inflamatorias y anti-hepatotóxicas (Lu et al., 2000). En el caso en estudio la sustancia MC28-03 no mostró actividad antibacteriana ni inhibitoria de la MAO-A y MAO-B, lo cual no implica que pueda contar con otro tipo de actividad biológica.

\section{CONCLUSIÓN}

En la Comunidad de Angochagua, el índice de diversidad de cultivos tiene un valor 2,4 siendo un valor alto comparado con otras zonas del planeta que oscila entre 1,5 y 2,0.

En relación con la información recopilada, de acuerdo con el sexo de las personas de la comunidad encuestadas se muestra que no existen diferencias significativas en el conocimiento de hombre y mujeres en el número de plantas de uso medicinal conocidas $(\mathrm{X} 2 \mathrm{cal}=0,001, \mathrm{X} 2$ th $=$ $2,74, \mathrm{gl}=1, \mathrm{p}=1,0)$.
Monnina crassifolia es la especie que más ha perdido el uso tradicional teniendo como resultado RVU: 0,15; UST: $10 \%$, siendo esta la especie a estudiar en cuanto a sus principios activos para revalorizar su conocimiento ancestral.

Se obtuvieron extractos en orden de polaridad ascendente de la raíz de la especie Monnina crassifolia. Los extractos obtenidos revelan la existencia de compuestos sobre todo de media y alta polaridad. 
A partir del extracto metanólico, por medio de cromatografía TLC preparativa fase reversa, se obtuvieron dos fracciones de peso $3,8 \mathrm{mg}$ y $2,4 \mathrm{mg}$, identificando por medio de los espectros de RMN de ${ }^{1} \mathrm{H}$ y ${ }^{13} \mathrm{C}$, que corresponden presumiblemente a una saponina triterpénica, con una fracción benzoato y otro acetato.

\section{LISTA DE REFERENCIAS}

Bashir, A., Hamburger, M., Gupta, M. P., Solis, P., \& Hostettmann, K. (1992). Biphenyls and a xanthone from Monnina sylvatica. Phytochemistry, 31(9), 3203-3205.

Calixto, J. B. (2005). Twenty-five years of research on medicinal plants in Latin America: a personal view. Journal of ethnopharmacology, 100(1), 131134.

Cioffi, G., Dal Piaz, F., De Caprariis, P., Sanogo, R., Marzocco, S., Autore, G., \& De Tommasi, N. (2006). Antiproliferative Triterpene Saponins from Entada africana $\ldots$. Journal of Natural Products, 69(9), 1323-1329.

Chivian, E. (2002). Biodiversity: its importance to human health. Center for Health and the Global Environment, Harvard Medical School, Cambridge, MA.

Eriksen, B. (1993c). A revision of Monnina subg. Pterocarya (Polygalaceae) in northwestern South America. Annals of the Missouri Botanical Garden, 191-207. 
Eriksen, B., \& Persson, C. (2007). Polygalaceae. Flowering Plants. Eudicots, 345363.

Fu, G.-M., Wang, Y.-H., Gao, S., Tang, M.-J., \& Yu, S.-S. (2005). Five new cytotoxic triterpenoid saponins from the roots of Symplocos chinensis. Planta medica, 71(07), 666-672.

Grondona, E. M. (1945). Las especies argentinas del género Monnina (Polygalaceae). Darwiniana, 7(1), 1-37.

Germosén-Robineau L. (1995) Hacia una farmacopea vegetal caribeña. Edición TRAMIL 7. Enda - Caribe, UAG \& Universidad de Antioquia. Santo Domingo; (4-5)

Hidalgo, P. (2017), Aislamiento e identificación de metabolitos secundarios a partir de la raíz de la especie Monnina crassifolia (Ivilán) (tesis de pregrado). Universidad Técnica Particular de Loja. Loja, Ecuador

Kinghorn, A. D. (1992). Plants as sources of medicinally and pharmaceutically important compounds Phytochemical Resources for Medicine and Agriculture (pp. 75-95): Springer.

Lepore, L., Malafronte, N., Condero, F. B., Gualtieri, M. J., Abdo, S., Dal Piaz, F., \& De Tommasi, N. (2011). Isolation and structural characterization of glycosides from an anti-angiogenic extract of Monnina obtusifolia HBK. Fitoterapia, 82(2), 178-183.

Lu, Y., Umeda, T., Yagi, A., Sakata, K., Chaudhuri, T., Ganguly, D., \& Sarma, S. (2000). Triterpenoid saponins from the roots of tea plant (Camellia sinensis var. assamica). Phytochemistry, 53(8), 941-946.

Mahmood, N., Moore, P., De Tommasi, N., De Simone, F., Colman, S., Hay, A., \& Pizza, C. (1993). Inhibition of HIV infection by caffeoylquinic acid derivatives. Antiviral Chemistry and Chemotherapy, 4(4), 235-240.

Olugbade, T. A., Ogundaini, A., Birlirakis, N., Païs, M., \& Martin, M.-T. (2000). Petersaponins III and IV, triterpenoid saponins from Petersianthus macrocarpus. Journal of Natural Products, 63(5), 716-719. 
Pinto, D. C., Fuzzati, N., Chiriboga, X., \& Hostettmann, K. (1994). Xanthone and antifungal constituents from Monnina obtusifolia. Phytochemistry, 37(3), 875-878.

Sandoval, H., (2017). Plan de desarrollo Territorial de la Parroquia de Angochagua. Ecuador, Gobierno Autònomo Desentralizado de la Parroquia Rural de Angichagua. 11-20.

Ranilla, L. G., Kwon, Y.-I., Apostolidis, E., \& Shetty, K. (2010). Phenolic compounds, antioxidant activity and in vitro inhibitory potential against key enzymes relevant for hyperglycemia and hypertension of commonly used medicinal plants, herbs and spices in Latin America. Bioresource technology, 101(12), 4676-4689.

Rao, S. R., \& Ravishankar, G. (2002). Plant cell cultures: chemical factories of secondary metabolites. Biotechnology advances, 20(2), 101-153.

Rates, S. M. K. (2001). Plants as source of drugs. Toxicon, 39(5), 603-613.

Ruiz, H., López, H. R., \& Pavón, J. (1798). Systema vegetabilium florae peruvianae et chilensis: Typis Gabrielis de Sancha. 